\title{
Effect of smoking and soft tissue release on risk of revision after total knee arthroplasty: a case- control study
}

\author{
Benedict U. Nwachukwu ${ }^{3}$, Ellen B. Gurary ${ }^{1,5}$, Vladislav Lerner ${ }^{4}$, Jamie E. Collins ${ }^{1}$, Thomas S. Thornhill' \\ Elena Losina ${ }^{1,2,5}$ and Jeffrey N. Katz ${ }^{1,2,6^{*}}$
}

\begin{abstract}
Background: Increasing utilization of primary total knee arthroplasty (TKA) is projected to expand demand for revision TKA. Revision TKAs are procedurally complex and incur high costs on our financially constrained healthcare system. The purpose of this study was to use a case-control design to identify factors predisposing to revision TKA, particularly demographic, clinical and perioperative technical factors.

Methods: We conducted a case control study to investigate patient, surgical and perioperative factors associated with greater risk of revision TKA. We included patients who received TKA at a tertiary center between 1996 and 2009. Cases (patients that had primary and revision TKA) were matched to controls (patients with primary TKA that was not revised) in a 1:2 ratio and risk of revision examined using conditional logistic regression.

Results: We identified 146 cases and 290 controls. Patient factors independently associated with revision included male sex (OR 1.73; $95 \% \mathrm{Cl} 1.06-2.81)$ and smoking (OR 2.87; 1.33-6.19). Older age was associated with decreased risk (OR 0.83 per 5-year increment; $95 \% \mathrm{Cl}$ 0.75-0.92). Lateral release was the only technical factor associated with revision (OR 1.92; 1.07-3.43).

Conclusions: In this case control study younger patient age, male gender, soft tissue release and active smoking status were associated with increased revision risk. Although we do not know whether the risk of smoking arises from short- or long-term exposure, smoking cessation prior to TKA should be considered as an intervention for decreasing revision risk.
\end{abstract}

\section{Background}

Total Knee Arthroplasty (TKA) is an effective surgical intervention for the relief of the symptoms and functional disability associated with advanced knee arthritis [1]. More than 610,000 primary total knee replacements were performed in 2012 incurring aggregate costs exceeding $\$ 9.8$ billion [2]. The increasing utilization of primary TKA is projected to result in substantial increases in demand for revision TKA, which was performed on over 54,000 persons in the US in 2012 [2]. Recent evidence suggests that the utilization of TKA is growing fastest in younger patients [3], a population at higher risk for revision procedures [4]. Given the procedural

\footnotetext{
*Correspondence: jnkatz@partners.org

'Department of Orthopedic Surgery, Boston, USA

${ }^{2}$ Division of Rheumatology, Immunology and Allergy, Boston, USA

Full list of author information is available at the end of the article
}

complexity of revision procedures, growth in utilization of revision TKAs will further strain healthcare resources [5].

The growing use of primary TKA and attendant burden of revision TKA have heightened interest in identifying risk factors for revision. Several factors have been reported to be associated with an elevated risk for revision TKA including male sex [6-8], younger age $[6,8]$, medical comorbidity [8], socioeconomic status [8], surgical technique $[9,10]$, body mass index (BMI) [11], postoperative knee alignment [11] and prior knee surgery [12]. The literature is limited, however, because many papers rely on administrative/registry databases $[13,14]$, which often do not have rich clinical detail to analyze for patient and technical factors associated with revision.

Given the limitations in the current evidence base, the purpose of this study was to use a case-control design to 
identify factors predisposing to revision TKA, particularly demographic, clinical and perioperative technical factors.

\section{Methods}

\section{Study design}

We conducted a case-control study of risk factors for revision TKA after primary TKA.

\section{Selection of patients \\ Patient population}

We identified subjects using the Partners Research Patient Data Registry (RPDR), a data repository that permits identification of all procedures performed at Brigham and Women's Hospital (BWH), Boston, MA USA using International Classification of Diseases Ninth Edition (ICD-9) and Current Procedural Terminology (CPT) codes. Patients were eligible for inclusion if they received a TKA between January 1996 and January 2009 at the BWH. The Institutional Review Board (IRB) at the BWH approved this study and the study design was exempt from the requirement for informed consent.

\section{Identification of cases and controls}

Patients were eligible for inclusion as cases if they underwent both primary TKA and subsequent revision TKA at BWH. We attempted to match each case with a control patient who received primary TKA at BWH and did not undergo revision TKA during the study period. We matched cases to controls in a 1:2 ratio based on primary surgeon and year of surgery. Matching by surgeon was done to eliminate the risk of revision attributable to surgeon factors and matching by year was done to minimize the impact of changing surgical techniques over the time period of the study.

\section{Chart review}

Following the identification of cases and controls we conducted a medical record review. We obtained medical record information from the RPDR, paper medical record or BWH electronic Longitudinal Medical Record (LMR). Specific data elements that we abstracted included: primary underlying joint disease, medical comorbidities (past medical history, medications, body mass index), prior orthopaedic and non-orthopaedic surgeries, socio-demographics (employment status, living conditions, marital status, ambulatory status and smoking status), perioperative factors (hospital length of stay, discharge destination, antibiotic treatment), surgical factors (anesthesia type, primary surgeon, surgical and tourniquet time, surgical approach, fixation type, ligament/ soft tissue releases), implant type and design, pre and post-operative radiographic alignment and immediate post-operative complications. We abstracted data on patient and technical factors for inclusion in analysis.
For subjects undergoing revision TKA the medical record review also included indication for revision, revision procedure performed, operative findings and primary surgeon.

\section{Statistical analysis}

We used conditional logistic regression for matched data to calculate univariate odds ratios characterizing the association between covariates and case/control status, and $95 \%$ confidence intervals (CI). We advanced factors associated with revision with $p$-value less than 0.1 or odds ratio greater than 1.5 or less than 0.75 from univariate analysis to multivariable conditional logistic regression analysis. Univariate associations are presented as crude odds ratios and multivariate associations as adjusted odds ratios based on the final model. Because there was some missing data on whether a lateral release was performed, we introduced an indicator variable "lateral release missing" to examine revision risk in subjects with missing data on lateral release and to avoid dropping these patients from the model. The sample size of 146 cases generally supports a multivariate analysis of 14 risk factors $[15,16]$. We performed a pair of sensitivity multivariate analyses in which we first restricted the sample to cases due to infection (and their controls) and then to cases due to aseptic revision (and their controls).

\section{Results}

Sample

Our study includes a total of 147 consecutive revision TKAs (cases). One case could not be matched with a control TKA and was excluded, leaving 146 cases in the sample. Of these cases, 144 had two controls and 2 could only be matched to one control yielding a total of 290 controls. Thus the final sample included 146 cases and 290 controls.

The mean age of the cases was 57.8 (sd.13.2) years and 65.4 (sd.12.6) years for the controls. The majority of cases $(N=91 ; 62 \%)$ and controls $(N=216 ; 74 \%)$ were female. Osteoarthritis $(\mathrm{OA})$ was the predominant indication for primary TKA in cases $(N=118 ; 84 \%)$ and controls $(N=266 ; 92 \%)$ (Table 1$)$.

Infection was the most common indication for revision $(N=44,30 \%)$, followed by aseptic loosening $(n=26$, $18 \%)$ and stiffness $(n=26,18 \%)$ and then instability $(n=$ $19,13 \%$ ) (Table 2).

\section{Univariate analysis of factors associated with revision}

In univariate analysis cases were more likely to be younger than controls (OR 0.79 per 5 years; $95 \%$ CI $0.72-$ 0.86). Males had a statistically significant increased risk for revision (OR 1.77; 95 \% CI 1.15-2.72). Receiving TKA for a non-OA diagnosis increased the risk of revision (OR 2.03; 95 \% CI 1.09-3.79). Twenty-nine cases 
Table 1 Case control comparison for risk of revision based on demographic and technical factors

\begin{tabular}{|c|c|c|c|c|}
\hline Variables & Cases $(N=146)$ & Controls $(N=290)$ & Crude odds ratios & Adjusted odds ratios- final model $^{a}$ \\
\hline Continuous & Mean (SD) & Mean (SD) & OR $(95 \% \mathrm{Cl})$ & OR $(95 \% \mathrm{Cl})$ \\
\hline Age at Surgery ${ }^{b}$ & $57.8 \pm 13.2$ & $65.4 \pm 12.6$ & $0.79(0.72,0.86)$ & $0.83(0.75,0.92)$ \\
\hline Cardiac Risk $(0,1,2+)^{c}$ & $1.6 \pm 0.7$ & $1.7 \pm 0.7$ & $0.91(0.67,1.22)$ & \\
\hline Categorical & N (\%) & N (\%) & OR $(95 \% \mathrm{Cl})$ & OR $(95 \% \mathrm{Cl})$ \\
\hline \multicolumn{5}{|l|}{ Gender } \\
\hline Male & 55 (38 \%) & $74(26 \%)$ & $1.77(1.15,2.72)$ & $1.73(1.06,2.81)$ \\
\hline Female & $91(62 \%)$ & $216(74 \%)$ & reference & reference \\
\hline \multicolumn{5}{|l|}{ Smoking } \\
\hline Yes & $29(22 \%)$ & $18(6 \%)$ & $4.46(2.21,9.03)$ & $2.87(1.33,6.19)$ \\
\hline No & 104 (78 \%) & $263(94 \%)$ & reference & reference \\
\hline \multicolumn{5}{|c|}{ OA Diagnosis at Primary } \\
\hline No & $22(16 \%)$ & $24(8 \%)$ & $2.03(1.09,3.79)$ & $1.55(0.75,3.22)$ \\
\hline Yes & 118 (84 \%) & 266 (92\%) & reference & Reference \\
\hline \multicolumn{5}{|l|}{ BMI Category } \\
\hline$<30$ & 75 (51 \%) & $153(53 \%)$ & reference & \\
\hline $30-35$ & $31(21 \%)$ & $70(24 \%)$ & $0.84(0.52,1.35)$ & \\
\hline $35+$ & $40(27 \%)$ & $67(23 \%)$ & $0.77(0.44,1.34)$ & \\
\hline \multicolumn{5}{|l|}{ Diabetes } \\
\hline Yes & $14(10 \%)$ & $43(15 \%)$ & $0.62(0.33,1.17)$ & \\
\hline No & 132 (90 \%) & $247(85 \%)$ & reference & \\
\hline \multicolumn{5}{|l|}{ PCL Recession } \\
\hline Yes & 19 (15 \%) & $51(19 \%)$ & $0.67(0.35,1.26)$ & \\
\hline No & 111 (85\%) & 212 (81 \%) & reference & \\
\hline \multicolumn{5}{|l|}{ Lateral Release } \\
\hline Yes & $42(29 \%)$ & $59(20 \%)$ & $1.85(1.13,3.01)$ & $1.92(1.07,3.43)$ \\
\hline No & $88(60 \%)$ & $216(75 \%)$ & Reference & Reference \\
\hline Missing & 16 (11 \%) & 15 (5 \%) & $2.61(1.24,5.47)$ & $1.68(0.68,4.13)$ \\
\hline \multicolumn{5}{|l|}{ Post-Op Return to OR } \\
\hline Yes & $22(15 \%)$ & 10 (3 \%) & $4.65(2.13,10.13)$ & \\
\hline No & 124 (85 \%) & 280 (97\%) & reference & \\
\hline
\end{tabular}

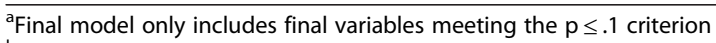

${ }^{b}$ Odds Ratios in units of 5 years

'The following were defined as cardiac risk factors: Coronary Artery (CAD); Coronary Artery Bypass Graft (CABG); Atherosclerotic Cardiovascular (ASCVD); Ischemic Heart Disease. Patients were assigned a score $(0,1,2+)$ based on a sum for each individual disease ( 0 if no disease and 1 if disease was present)

(22\%) and 18 controls (6\%) were current smokers. Active smoking status was significantly associated with revision (OR 4.46; 95 \% CI 2.21-9.03) (Table 1). We did not observe statistically significant associations between revision and cardiovascular comorbidity, diabetes, preoperative varus/valgus malalignment and pre-operative ambulatory status.

With regard to technical factors, intra-operatively, $29 \%$ of cases received a lateral release at the time of primary TKA compared to $20 \%$ of controls. The use of a lateral release significantly increased the risk of revision (OR 1.85; 1.13-3.01). Cases were also more likely to have missing data on lateral release than controls (Table 1).
Further analysis showed that of the 42 cases that had lateral release, 18 (46\%) were revised for infection. PCL technique (sparing/sacrifice) did not have a statistically significant association with revision risk.

Factors that were not associated with revision risk (at $p<0.05$ or $0.75<\mathrm{OR}<1.5)$ are not shown on Table 2.

Multivariate analysis of factors associated with revision We performed multivariate, conditional logistic regressions analyses, advancing all variables with univariate $p$ values $<0.1$ or odds ratios $>1.5$ or $<0.75$. The final model is shown in Table 1. We did not advance return to $\mathrm{OR}$ to the final model because information on return 
Table 2 Indications for revision ${ }^{\mathrm{a}}$

\begin{tabular}{ll}
\hline Causes of Failure & Total \\
\hline Aseptic Loosening & N=146 \\
Extensor Mechanism Failure & $26(18 \%)$ \\
Infection & $44(30 \%)$ \\
Instability & $19(13 \%)$ \\
Periprosthetic Fracture & $2(1 \%)$ \\
Polyethylene Liner Wear & $1(1 \%)$ \\
Repeated Dislocation & $3(2 \%)$ \\
Stiffness & $26(18 \%)$ \\
Subsidence & $2(1 \%)$ \\
Swelling & $10(7 \%)$ \\
Other & $11(8 \%)$ \\
\hline
\end{tabular}

Indications listed hierarchically. If subject had more than one indication, he or she was assigned the one highest on this list

to OR is not known preoperatively and therefore cannot be incorporated into preoperative planning. In our final model increased age was associated with decreased revision risk (OR 0.83 per 5 years; $95 \%$ CI 0.75-0.92); male sex (OR 1.73; 1.06-2.81) and current smoking status (OR 2.87 ; $1.33-6.19)$ were associated with increased revision risk. Lateral release (OR 1.92; 1.07-3.43) was associated with an increased risk of revision and having missing data on lateral release was also associated with an increased risk of revision, although this association did not reach statistical significance (OR 1.68, 0.68, 4.13).

We performed exploratory analyses in which we examined risk factors for revision due to infection and risk factors for aseptic revision in separate models. In these models, lateral release was associated strongly with risk of revision for infection (OR 5.28, 95 \% CI 1.69, 16.47) but the data did not provide evidence for a clinically important relationship of lateral release with aseptic revision (OR 1.28, 95 \% CI 0.60, 2.74). In contrast, smoking was associated strongly with risk of aseptic revision (OR $4.41,95 \%$ CI 1.67, 11.62) but the data did not support a clinically important relationship of smoking with risk for infectious revision (OR 1.2295 \% CI 0.23, 6.64). As in the models of the entire cohort, the adjusted ORs for the group of subjects missing data on lateral release were similar to those for lateral release.

\section{Discussion}

We performed a case control study to investigate factors associated with revision TKA at a tertiary care center. Patient factors increasing the risk for revision included younger age, male gender and smoking status. Technical factors associated with increased revision risk included receiving a lateral release.
Our finding that males and younger patients have an increased risk for revision is a consistent finding in the literature and has been reproduced in large database studies [13, 8]. Curtin et al. [8] found, based on analysis of 61,767 TKAs, that younger male patients were more likely to require a revision procedure after primary TKA. This phenomenon is likely explained by greater stresses placed on the implant by young active male patients and potentially greater reluctance among clinicians to revise a TKA in older patients.

We also found that smoking status has a strong association with revision risk for TKA. While smoking has been associated with an increased risk of general postoperative complication after TKA [17-19], only one previous study has specifically examined the association between revision TKA and smoking status [20]. Kapadia et al. performed a survivorship analysis of TKAs performed on 531 patients at a mean follow-up of 47 months and found that any history of smoking (current or former) was associated with a higher revision rate (10 \% in smokers vs $1 \%$ in non-smokers). Our finding in a study with longer follow up and controlling for potential confounders confirms this association. Although the pathophysiology underlying the increased risk for revision TKA in smokers has not been clearly elucidated, cigarette smoke has been shown to disrupt osteointegration, a fundamental process in the longevity of implanted materials [21]. In addition, nicotine has been found to inhibit secretion of tumor necrosis factor (TNF) alpha-a critical cytokine for bone remodeling-through activation of the cholingergic anti-inflammatory pathway [22]. Previous evidence has shown that smoking cessation programs can attenuate the general surgical risk associated with active smoking status [23]. More attention should be paid to understanding the impact of smoking cessation on revision risk after TKA.

There is increasing interest in understanding the technical factors associated with revision TKA. In this study we found that performing a lateral release increased the risk for a revision TKA. Lateral release during TKA is a surgical technique whereby tight lateral soft tissue structures of the knee are released. Such releases are most often employed in valgus knees in order to balance the knee and restore joint kinematics. The impact of lateral release during TKA has been previously studied. Patellofemoral maltracking and patella osteonecrosis/fracture are potential biomechanical complications associated with lateral release. However there has been evidence to suggest that with appropriate surgical technique these complications are avoidable and do not increase revision risk [24, 25]. Similar to these prior studies our subgroup analysis did not find an increased incidence of instability or loosening in patients requiring a lateral release. We did find however that lateral release was associated with 
increased risk for infection related revision. A few prior studies have suggested that there is potentially an association between soft tissue releases and increased infection risk after TKA. Kumar and Dorr [26] hypothesized that in valgus knees requiring soft tissue release there is an increased risk for infection due to the creation of irregular tissue planes and dead space, which may serve as a nidus for infection. Johnson and Eastwood [27] similarly found that when the superolateral geniculate vessels are divided during lateral release there is an increased risk of wound infection and skin viability as assessed by skin oxygen tension. Lateral releases are an important part of knee balancing during TKA and should continue to be utilized when indicated however continued attention should be paid to understanding the risk factors for revision and the role of lateral releases. The risk for revision noted in the present study for lateral release is likely separate from that associated with having valgus alignment alone, since valgus alignment was not associated with revision risk in our analyses.

This study has several limitations. Our case control match was based on surgeon and year of primary procedure. Matching on surgeon precludes analysis of surgeon factors that may play a role in revision risk (e.g. arthroplasty fellowship training or surgeon volume). As part of this study we looked at the impact of preoperative alignment however we did not look at the severity and degree of knee malalignment on revision risk; this may have modulated the impact of lateral release. Further as part of our dataset, operative information on lateral release was missing in a select number of our cases; this may have influenced our findings. Our assessment of smoking status in this study was also limited. We were unable to obtain information on pack-years and thus cannot comment on dose response or the effect of smoking cessation on revision risk. We also did not have data on alcohol or illicit drug use. Finally, previous studies have suggested that social and ethnic factors contribute to TKA outcome [28]. Due to inconsistent reporting of race and socioeconomic variables such as income or education within the database, and lack of information on payor status, we were not able to sufficiently track these variables to use in this study.

The major strength of this study is that we performed detailed medical record reviews. We used a detailed review of institutional medical records, with outpatient, in-patient and intra-operative data. This approach enabled us to identify factors such as smoking status and lateral release that generally cannot be determined from large administrative medical datasets but which appear to influence revision risk. More work needs to be done to understand the impact of lateral release on revision risk. Intra-operative lateral release may be indicative of other factors associated with increased likelihood for revision. For example, requiring a lateral release may be indicative of procedural complexity. Similarly, technical errors such as excessive femoro-tibial rotation may necessitate a lateral release, certain implants are associated with a higher incidence of lateral release and certain surgeons may perform these releases more commonly.

\section{Conclusion}

In this case control study younger patient age, male gender, soft tissue release and active smoking status were associated with increased revision risk. Although we do not know whether the risk of smoking arises from shortor long-term exposure, smoking cessation prior to TKA should be considered as an intervention for decreasing revision risk. Lateral release is a technical factor associated with increased risk for revision after TKA.

\section{Abbreviations}

BMl: Body mass index; OA: Osteoarthritis; TKA: Total knee arthroplasty.

\section{Competing interests}

The authors declare no competing interests. None of the authors has financial and/or personal relationships with other people or organizations that could potentially and inappropriately influence our work and conclusions.

\section{Authors' contributions}

BUN was involved in study design, data analysis and interpretation, writing the manuscript and critical revision of the manuscript. EBG was involved in critical revision of the manuscript, statistical expertise and performing most of the analyses presented in the paper. VL was involved in study conception and design, data collection, development of data collection instruments and critical revision of the manuscript. JEC was involved in data collection, preliminary data analysis and interpretation, critical revision of the manuscript and statistical expertise. TST was involved in the conception and design of the study, analysis and interpretation of data, critical revision of the manuscript and supervision and administrative support. EL was involved in study conception and design, data analysis and interpretation, critical revision of the manuscript. JNK was involved in study conception and design, data analysis and interpretation, writing the manuscript, critical revision of the manuscript, supervision and administrative support. All authors approved the final manuscript.

\section{Acknowledgment}

This work was supported by the Pilot Research Incubation and Development Program (PRIDE) through the Department of Orthopaedic Surgery at Brigham \& Women's Hospital as well as NIAMS T32AR055885.

\section{Author details}

${ }^{1}$ Department of Orthopedic Surgery, Boston, USA. ${ }^{2}$ Division of Rheumatology, Immunology and Allergy, Boston, USA. ${ }^{3}$ The Department of Orthopedic Surgery, Hospital for Special Surgery, New York, USA.

${ }^{4}$ Department of Orthopedic Surgery, Massachusetts General Hospital, Boston, USA. ${ }^{5}$ Department of Biostatistics, Boston University School of Public Health, Boston, USA. ${ }^{6}$ Orthopedic and Arthritis Center for Outcomes Research, Brigham and Women's Hospital, 75 Francis Street, BC-4016, Boston, MA 02115, USA.

Received: 6 March 2015 Accepted: 21 August 2015

Published online: 09 September 2015

\section{References}

1. Carr AJ, Robertsson O, Graves S, Price AJ, Arden NK, Judge A, et al. Knee replacement. Lancet. 2012;379(9823):1331-40. 
2. Healthcare Cost and Utilization Project (HCUP). Nationwide Inpatient Sample (NIS). Agency for Healthcare Research and Quality. http:// hcupnet.ahrq.gov/HCUPnet.jsp. Accessed May 182013.

3. Losina E, Thornhill TS, Rome BN, Wright J, Katz JN. The dramatic increase in total knee replacement utilization rates in the United States cannot be fully explained by growth in population size and the obesity epidemic. J Bone Joint Surg Am. 2012;94(3):201-7.

4. Kurtz SM, Lau E, Ong K, Zhao K, Kelly M, Bozic KJ. Future young patient demand for primary and revision joint replacement: national projections from 2010 to 2030. Clin Orthop Relat Res. 2009;467(10):2606-12.

5. Ong KL, Mowat FS, Chan N, Lau E, Halpern MT, Kurtz SM. Economic burden of revision hip and knee arthroplasty in Medicare enrollees. Clin Orthop Relat Res. 2006;446:22-8.

6. Heck DA, Melfi CA, Mamlin LA, Katz BP, Arthur DS, Dittus RS, et al. Revision rates after knee replacement in the United States. Med Care. 1998;36(5):661-9.

7. Robertsson O, Knutson K, Lewold S, Lidgren L. The Swedish Knee Arthroplasty Register 1975-1997: an update with special emphasis on 41,223 knees operated on in 1988-1997. Acta Orthop Scand. 2001;72(5):503-13.

8. Curtin B, Malkani A, Lau E, Kurtz S, Ong K. Revision after total knee arthroplasty and unicompartmental knee arthroplasty in the medicare population. J Arthroplasty. 2012;27(8):1480-6.

9. Barrack RL, Barnes CL, Burnett RS, Miller D, Clohisy JC, Maloney WJ. Minimal incision surgery as a risk factor for early failure of total knee arthroplasty. J Arthroplasty. 2009;24(4):489-98.

10. Abdel MP, Morrey ME, Jensen MR, Morrey BF. Increased long-term survival of posterior cruciate-retaining versus posterior cruciate-stabilizing total knee replacements. J Bone Joint Surg Am. 2011;93(22):2072-8.

11. Ritter MA, Davis KE, Meding JB, Pierson JL, Berend ME, Malinzak RA. The effect of alignment and BMI on failure of total knee replacement. J Bone Joint Surg Am. 2011;93(17):1588-96.

12. Suzuki G, Saito S, Ishii T, Motojima S, Tokuhashi Y, Ryu J. Previous fracture surgery is a major risk factor of infection after total knee arthroplasty. Knee Surg Sports Traumatol Arthrosc. 2011;19(12):2040-4.

13. Dy CJ, Marx RG, Bozic KJ, Pan TJ, Padgett DE, Lyman S. Risk factors for revision within 10 years of total knee arthroplasty. Clin Orthop Relat Res. 2014;472(4):1198-207. doi:10.1007/s11999-013-3416-6.

14. Namba RS, Cafri G, Khatod M, Inacio MC, Brox TW, Paxton EW. Risk factors for total knee arthroplasty aseptic revision. J Arthroplasty. 2013;28(8 Suppl):122-7. doi:10.1016/j.arth.2013.04.050.

15. Concato J, Peduzzi P, Holford TR, Feinstein AR. Importance of events per independent variable in proportional hazards analysis. I. Background, goals, and general strategy. J Clin Epidemiol. 1995;48(12):1495-501.

16. Vittinghoff $E, M c C u l l o c h ~ C E$. Relaxing the rule of ten events per variable in logistic and Cox regression. Am J Epidemiol. 2007;165(6):710-8. doi:10.1093/ aje/kwk052.

17. Peersman G, Laskin R, Davis J, Peterson M. Infection in total knee replacement: a retrospective review of 6489 total knee replacements. Clin Orthop Relat Res. 2001;392:15-23.

18. Møller AM, Pedersen T, Villebro N, Munksgaard A. Effect of smoking on early complications after elective orthopaedic surgery. J Bone Joint Surg (Br). 2003;85(2):178-81.

19. Sharrock NE, Hargett MJ, Urquhart B, Peterson MG, Ranawat C, Insall J, et al. Factors affecting deep vein thrombosis rate following total knee arthroplasty under epidural anesthesia. J Arthroplasty. 1993;8(2):133-9.

20. Kapadia BH, Johnson AJ, Naziri Q, Mont MA, Delanois RE, Bonutti PM. Increased revision rates after total knee arthroplasty in patients who smoke. J Arthroplasty. 2012;27(9):1690-5.

21. Fini M, Giavaresi G, Salamanna F, Veronesi F, Martini L, De Mattei M, et al. Harmful lifestyles on orthopedic implantation surgery: a descriptive review on alcohol and tobacco use. J Bone Miner Metab. 2011;29(6):633-44.

22. Chen Y, Guo Q, Pan X, Qin L, Zhang P. Smoking and impaired bone healing: will activation of cholinergic anti-inflammatory pathway be the bridge? Int Orthop. 2011;35(9):1267-70.

23. Thomsen T, Villebro N, Moller AM. Interventions for preoperative smoking cessation. Cochrane Database Syst Rev. 2010 July 7; (7):CD002294 doi: 10.1002/ 14651858.CD002294.pub3.

24. Weber AB, Worland RL, Jessup DE, Van Bowen J, Keenan J. The consequences of lateral release in total knee replacement: a review of over 1000 knees with follow up between 5 and 11 years. Knee. 2003;10(2):187-91.
25. Lachiewicz PF, Soileau ES. Patella maltracking in posterior-stabilized total knee arthroplasty. Clin Orthop Relat Res. 2006;452:155-8. doi:10.1097/ 01.blo.0000238803.97713.7d.

26. Kumar PJ, Dorr LD. Severe malalignment and soft-tissue imbalance in total knee arthroplasty. Am J Knee Surg. 1997;10(1):36-41.

27. Johnson DP, Eastwood DM. Lateral patellar release in knee arthroplasty. Effect on wound healing. The Journal of arthroplasty. 1992;7 (Suppl 1):427-31.

28. Nwachukwu BU, Kenny AD, Losina E, Chibnik LB, Katz JN. Complications for racial and ethnic minority groups after total hip and knee replacement: a review of the literature. J Bone Joint Surg Am. 2010;92(2):338-45.

\section{Submit your next manuscript to BioMed Central and take full advantage of:}

- Convenient online submission

- Thorough peer review

- No space constraints or color figure charges

- Immediate publication on acceptance

- Inclusion in PubMed, CAS, Scopus and Google Scholar

- Research which is freely available for redistribution 